\title{
Aspergillus Infection in the Ascending Aorta of a Patient with Aortic and Mitral Valve Prostheses
}

\author{
Auristela de Oliveira Ramos, Amira Rose Costa Medeiros, Paulo Paredes Paulista, Cely Saad Abboud, \\ Zilda Machado Meneghelo \\ São Paulo, SP - Brazil
}

We report the case of implantation of metallic mitral and aortic valve prostheses 6 months earlier, with subsequent multiple embolic episodes. The anatomicopathological examination of the thrombus of the third embolic episode was compatible with Aspergillus sp, which was treated with amphotericin $B$, followed by oral itraconazole. On the fourth embolism, vegetations were visualized in the ascending aorta on echocardiography and resonance imaging, and the patient underwent replacement of the aortic segment by a Haemashield tube and exploration of the aortic prosthesis, which was preserved, because no signs of endocarditis were found. Four months later, the patient died due to cardiogenic shock secondary to acute myocardial infarction caused by probable coronary embolism and partial dysfunction of the aortic prosthesis.

Although fungal infections occur more frequently in immunocompromised patients, several cases of this type of infection after cardiac surgery have been reported in the medical literature ${ }^{1,2}$. The incidence of infective endocarditis in valvar prostheses ranges from 7 to $25 \%{ }^{3-5}$. The higherrisk period comprises the 6 months following valvar replacement, with an incidence of infective endocarditis ranging from 1.4 to $3.1 \%$ in the first year and decreasing to $0.35 \%$ after 1 year. The most common infective agents in the first 60 days following prosthesis implantation are Staphylococcus aureus, the gram negative bacilli, the diphtheroids, and fungi. Fungi may account for up to $10 \%$ of the cases, with a predominance of Candida albicans and Aspergillus $s p^{3,6}$. The major predisposing factors for fungal infection are cardiac surgery, the use of intravenous drugs, prolonged intravenous antibiotic therapy, parenteral nutrition, and

Instituto Dante Pazzanese de Cardiologia

Mailing address: Auristela de Oliveira Ramos - Av. Miruna, 327/44 - 04084-001

- São Paulo, SP, Brazil - E-mail: rafframos@uol.com.br

English version by Stela Maris C. e Gandour severe immunosuppression ${ }^{1-4}$. Systemic embolism, predominating in the brain, is a frequent complication in this type of infection and occurs in 22 to $50 \%$ of the cases ${ }^{5}$.

\section{Case report}

The patient was a 27-year-old white male, plumber, from the Brazilian State of Ceara, residing in the city of São Paulo, who underwent implantation of 2 metallic prostheses on $2 / 14 / 01$. Six months later, the patient experienced an episode of acute arterial embolism in the left lower limb. The patient denied having fever and used oral anticoagulant agents regularly. His international normalized ratio was 3.0, and he underwent left infrapatellar popliteal thromboembolectomy. The thrombi had the gross appearance of clots and were not sent for anatomicopathological examination. The transesophageal echocardiography showed normofunctioning prostheses, spontaneous contrast medium (degree II) in the left atrium, and no intracavitary thrombi. After hospital discharge, the patient experienced a new episode of peripheral embolism and the anatomicopathological examination of the removed thrombi was compatible with mixed thrombi, focally organized, and negative for culture. The echocardiogram showed a sessile image adhered to the ascending aorta wall and projecting to the vessel lumen $1 \mathrm{~cm}$ away from the ring, suggestive of thrombosis. Anticoagulation was intensified and an antiplatelet agent was added. Three months later, the patient was hospitalized again with findings of arterial ischemia, an international normalized ratio of 5.4, and the anatomicopathological examination of the embolus showed fungal infection in recent thrombi, whose morphology was compatible with Aspergillus sp. The transesophageal echocardiogram showed normofunctioning prostheses and an image in the ascending aorta similar to that previously reported. The hemogram was as follows: hematocrit, $30 \%$; hemoglobin, $10 \mathrm{~g} / \mathrm{L}$; leukocytes, 9000 , with no left shift; hemosedimentation rate, $100 \mathrm{~mm} / 1 \mathrm{st}$ hour; C-reactive protein, $10 \mathrm{mg} / \mathrm{dL}$; and negative anti-HIV 
serology. Tomographies of the brain, chest, and abdomen were performed in the search for aspergilloma and resulted in negative findings. Therapy with intravenous amphotericin B was initiated and followed by oral itraconazole (100 mg per day) until completing 90 days of treatment. One month after the beginning of the treatment, the patient experienced the fourth peripheral embolic phenomenon. The transesophageal echocardiogram showed large vegetations in the ascending aorta, $1.0 \mathrm{~cm}$ above the prosthesis. Angioresonance of the thoracic and abdominal aorta showed an image of a vegetating, pendunculated mass of irregular contours in the anterior aortic wall directly above the valvar plane, with an approximate extension of $5 \mathrm{~cm}$ and not involving the supraaortic branches (fig. 1). The descending and abdominal aorta showed no alterations. The blood cultures persisted as negative. Intravenous antifungal therapy with amphotericin B was reinitiated and surgery was performed, showing large fungal vegetations directly above the valvar plane. The aortic and mitral prostheses were normofunctioning and covered with apparently normal endocardium, and their suture rings were free of thrombi and vegetation, therefore, they were preserved. The ascending aorta was sectioned below the brachiocephalic arterial trunk, and a supracoronary Haemashield (number 24) straight tube was implanted. The patient continued to take amphotericin B, completing 90 days of intravenous treatment, followed by oral itraconazole $(100 \mathrm{mg})$ for 90 more days, to complete the total 6-month treatment (intravenous and oral routes). On 5/17/02, the patient died in the emergency department with the diagnosis of cardiogenic shock. The electrocardiogram was compatible with extensive anterior infarction, and troponin I was $5.8 \mathrm{ng} / \mathrm{mL}$. The echocardiogram showed an image of vegetation in the aortic prosthesis with dysfunction and extensive anterior hypokinesia.

\section{Discussion}

After Candida, Aspergillus sp is the major cause of fungal infection in cardiac valves and prostheses and in the great vessels. Aspergillus fumigatus and flavus are the most common species. The incidence of fungal infection after im-

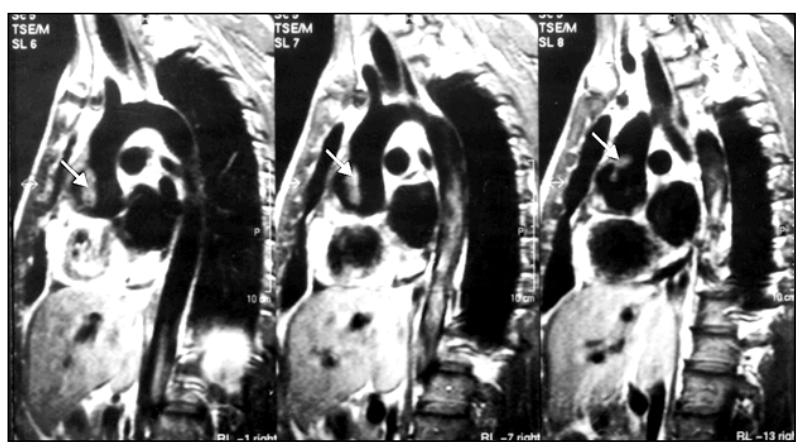

Fig. 1 - Angioresonance of the aorta (sagittal views) showing a vegetating pendunculated (arrows) image involving the ascending aorta.

plantation of a cardiac prosthesis is low, lower than $0.1 \%$ per year. The clinical findings may be similar to those of other types of infection; however, the presence of great valvar or prostheses destruction, large vegetations, and embolic phenomena, draw attention to the presence of the fungus. Clinical and pathological evidence is necessary for diagnostic confirmation, because blood cultures are rarely positive.

Treatment is based on the administration of antifungal agents for a long period and on early surgical intervention. The medication of choice is intravenous amphotericin B. In case of a good therapeutical response, amphotericin B can be replaced after 2 to 3 weeks by oral itraconazole to complete 90 days of treatment. Although cases of isolated medicamentous treatment for this type of infection exist, the best therapeutic strategy has been its association with surgical treatment. Before 1969, no patient with fungal endocarditis survived. Currently, mortality continues to be high. Survival of $67 \%$ in a 52-month follow-up has been reported in a small sample of patients with endocarditis due to Candida. Most problems are due to diagnostic difficulty and the consequent delay in specific treatment.

In the case here reported, the fungal infection was noteworthy, affecting initially the aorta but not the prostheses. The patient had poor clinical findings, no deterioration in his general condition or hyperthermia during the entire hospitalization, was not immunocompromised, and did not have the epidemiology for that type of infection. Surgery for prosthesis implantation may have been the entry of the infection.

\section{References}

1. Verghese S, Mullasari A, Padmaja P, et al. Fungal endocarditis cardiac surgery. Indian Heart J 1998; 50: 418-22.

2. Escribano Subias P, Lopez Rios F, Delagado Jimenez JF, et al. Mycotic aneurysm caused by Aspergillus of aortic suture line after heart transplantation. Rev Esp Cardiol 2000; 53:1403-5

3. Mylonakis E, Calderwood SB. Infective endocarditits in adults. N Engl J Med 2001; 345: 1318-29
4. MenesalvasA, Bouza E. Infective endocarditis caused by unusual microorganisms. Rev Esp Cardiol 1998; 51 (suppl 2): 79-85

5. Bayer AS, Bolger Af, Taubert KA, et al. Diagnosis and manegement of infective endocarditis and its complications. Circulation 1998; 2936-48.

6. Koshi G, Cherian KM. Aspergillus terreus, an uncommon fungus causing aortic root abscess and pseudoaneurysm. Indian Heart J 1995; 47: 265-7. 